\title{
BV and non-BV associated Gardnerella vaginalis establish similar synergistic interactions with other BV-associated microorganisms in dual-species biofilms
}

\author{
Joana Castro ${ }^{\mathrm{a}, \mathrm{b}}$, Nuno Cerca ${ }^{\mathrm{a}, *}$ \\ ${ }^{a}$ Centre of Biological Engineering (CEB), Laboratory of Research in Biofilms Rosário Oliveira (LIBRO), University of Minho, Campus de Gualtar, \\ 4710-057 Braga, Portugal \\ ${ }^{\mathrm{b}}$ Instituto de Ciências Biomédicas Abel Salazar (ICBAS), University of Porto, Rua de Jorge Viterbo Ferreira 228, 4050-313 Porto, Portugal
}

\section{A R T I C L E I N F O}

\section{Article history:}

Received 5 October 2015

Received in revised form

19 October 2015

Accepted 21 October 2015

Available online 24 October 2015

\begin{abstract}
A B S T R A C T
Dual-species biofilm formation between Gardnerella vaginalis strains isolated from women with or without bacterial vaginosis (BV) and other $24 \mathrm{BV}$-associated microorganisms support that the key difference in virulence potential between BV-negative and BV-positive G. vaginalis strains seems not to be related with biofilm maturation.
\end{abstract}

() 2015 Elsevier Ltd. All rights reserved.

Keywords:

Polymicrobial biofilms

Bacterial vaginosis

Gardnerella vaginalis virulence

Bacterial vaginosis (BV) is often characterized by a shift of the vaginal microbiota from a Lactobacillus-dominated community to a dense biofilm containing a complex mixture of microorganisms [1]. Gardnerella vaginalis is the dominant pathogen colonising BV women, often adopting the biofilm mode of growth as a survival strategy [2]. During BV, there is a complex interplay between pathogenic species, endogenous vaginal microbiota and the vaginal epithelium $[1,3,4]$. These interactions become more complex when microbes are adhered to the epithelium, forming biofilms, and communicate via "quorum-sensing", a cell-density dependent bacterial intercellular signalling mechanism [5]. However, G. vaginalis can also be a part of the vaginal microbiota in healthy women [6]. This raised the question whether there are pathogenic and commensal lineages within this species. Jayaprakash and colleagues provided genomic evidence that all G. vaginalis strains had the potential to form biofilm but not all strains had the potential to cause BV symptoms, namely due to absence of sialidase gene [7]. We recently also provided in vitro evidence that supports Jayaprakash hypothesis [8]. However, only the BV isolates demonstrated higher cytotoxicity and were able to adhere in high density clusters to a HeLa cell line [9], a condition necessary to foster in vivo biofilm

\footnotetext{
* Corresponding author.

E-mail address: nunocerca@ceb.uminho.pt (N. Cerca)
}

development [5]. Another important insight providing evidence that not all G. vaginalis have the same virulence potential was derived from recent in vivo observations by Swidsinski and colleagues. They demonstrated the presence of adherent bacterial biofilms in $90 \%$ of biopsies from women with BV, while only $10 \%$ of healthy women exhibited a similar biofilms [10]. Subsequently, they proposed that the mere presence of loosely adherent $G$. vaginalis on the vaginal epithelium was of lesser clinical significance than the presence of high density clusters of $G$. vaginalis [2]. In effort to better understand the differences between virulent and non-virulent $G$. vaginalis strains, the aim of the present study was to analyze the interactions between non-BV $(n=3)$ or $\mathrm{BV}(n=3)$ $G$. vaginalis isolates and other $\mathrm{BV}$-associated pathogens $(n=24)$ using a dual-species biofilm assembly, consisting in the combination of G. vaginalis and secondary BV-associated species. All species used are listed in Table 1.

The dual-species biofilm formation model used was the same as described by Machado and colleagues [11], with some minor modifications. Briefly, G. vaginalis cultures were adjusted to $1 \times 10^{7}$ colony-forming units (cfu)/mL by optical density (OD) at $600 \mathrm{~nm}$ (Model Sunrise, Tecan). After homogenization, $100 \mu \mathrm{L}$ of each bacterial suspension of $G$. vaginalis isolates was dispensed into each well of 96-well flat-bottom tissue culture plate (Orange Scientific). The tissue cultured plates were then placed in an incubator 
Table 1

GenBank accession numbers of strains used in this study.

\begin{tabular}{|c|c|c|}
\hline Bacteria $^{a, b}$ & Genes & Accession numbers ${ }^{c}$ \\
\hline Actinomyces neuii UM067An & $16 S$ rRNA & KT805271 \\
\hline Actinomyces turicensis UM066At & $16 S$ rRNA & KT805270 \\
\hline Aerococcus christensenii UM137Ac & $16 S$ rRNA & KT805273 \\
\hline Bacillus firmus UM034Bf & $16 S$ rRNA & KT805263 \\
\hline Brevibacterium ravenspurgense $\mathrm{UM} 066 \mathrm{Br}$ & $16 S$ rRNA & KT805269 \\
\hline Corynebacterium amycolatum UM065Ca & $16 S \mathrm{rRNA} / \mathrm{rpoB}$ & КT805275/КT923481 \\
\hline Corynebacterium tuberculostearicum UM137Ct2 & $16 S$ rRNA/rpoB & КТ805279/КТ923486 \\
\hline Corynebacterium tuscaniense UM137Ct & $16 S \mathrm{rRNA} / \mathrm{rpoB}$ & КT805278/KT923485 \\
\hline Enterococcus faecalis UM035 & $16 S$ rRNA & KT614045 \\
\hline Escherichia coli UM056 & $16 S$ rRNA & KT614048 \\
\hline Gardnerella vaginalis UM085 & $16 S$ rRNA & КР996679 \\
\hline Gardnerella vaginalis UM121 & $16 S$ rRNA & KP996681 \\
\hline Gardnerella vaginalis UM131 & $16 S$ rRNA & КР996676 \\
\hline Gardnerella vaginalis UM137 & $16 S$ rRNA & КР996682 \\
\hline Gardnerella vaginalis UM241 & $16 S$ rRNA & КР996683 \\
\hline Gardnerella vaginalis UM246 & $16 S$ rRNA & КР996677 \\
\hline Gemella haemolysans UM034Gh & $16 S$ rRNA & KT805264 \\
\hline Lactobacillus vaginalis UM062Lv & $16 S$ rRNA & KT805268 \\
\hline Mobiluncus mulieris ATCC 35239 & whole genome & NZ_GL405260.1 \\
\hline Nosocomiicoccus ampullae UM121Na & $16 S$ rRNA & KT805272 \\
\hline Prevotella bivia ATCC 29303 & $16 S$ rRNA & L16475.1 \\
\hline Propionibacterium acnes UM034Pa & $16 S$ rRNA & KT805265 \\
\hline Streptococcus agalactiae UM035Sa & $16 S$ rRNA & KT805266 \\
\hline Staphylococcus epidermidis UM066Se & $16 S \mathrm{rRNA} / \mathrm{rpoB}$ & КТ805277/КТ923483 \\
\hline Staphylococcus haemolyticus UM066Sh & $16 S \mathrm{rRNA} / \mathrm{rpoB}$ & КТ805276/КТ923482 \\
\hline Staphylococcus hominis UM224Sh & rрoв & КT923487 \\
\hline Staphylococcus saprophyticus UM121Ss & rpoB & KT923484 \\
\hline Staphylococcus simulans UM059Ss & $16 S$ rRNA & KT805267 \\
\hline Staphylococcus warnerii UM224Sw & rров & КТ923488 \\
\hline Streptococcus anginosus UM241b & $16 S$ rRNA & KT805274 \\
\hline
\end{tabular}

at $37{ }^{\circ} \mathrm{C}$ in $10 \% \mathrm{CO}_{2}$. Following $24 \mathrm{~h}$, the culture medium covering the biofilm was carefully removed and replaced by fresh medium. A second inoculation with $1 \times 10^{7} \mathrm{cfu} / \mathrm{mL}$ of each BV-associated strain was performed and biofilms were allowed to growth for another 24 h. Quantification of biofilm was performed by the crystal violet staining, as previously described [12]. All assays were repeated at least 3 times with 8 technical replicates. The data were analyzed using the non-parametric Kruskal-Wallis test, since the data did not follow a normal distribution according Kolmogorov-Smirvon's test, with the statistical software package SPSS 17.0 (SPSS Inc. Chicago, IL). $P$-values of less than 0.05 were considered significant.

As described in Fig. 1, our results revealed that $54 \%(n=13)$ of the BV-associated species tested had a synergistic effect in most of G. vaginalis strains. However, only 6 species caused an increase in biofilm formation in all tested conditions: Actinomyces neuii, Brevibacterium ravenspurgense, Corynebacterium amycolatum, Corynebacterium tuscaniense, Staphylococcus hominis and Staphylococcus saprohyticus. Conversely, we observed that $42 \%$ of the tested species showed variable interactions dependent of the specific $G$. vaginalis strain used. However, no link ( $P=0.131$; Kruskal-Wallis) was found between non-BV and BV G. vaginalis strains, with the exception of Mobiluncus mulieris, which showed an antagonistic effect when added to the biofilm formed by BV strains, whereas a synergistic interaction was verified in presence biofilms formed by non-BV G. vaginalis isolates. Finally, our data also revealed an antagonistic interaction between all G. vaginalis strains tested and Lactobacillus vaginalis.

The most recent model for the pathogenesis of BV suggests that
G. vaginalis adhered to vaginal epithelium might be acting as a scaffold for the attachment of a subsequent species [1,13]. However, the role of BV-associated bacteria in multi-species biofilms is still poorly understood. An early study by Machado and colleagues demonstrated that a few secondary BV-associated anaerobes, such as Prevotella bivia, were able to increment the concentration of cells within the biofilm, when added to a pre-formed G. vaginalis biofilms [11].

Herein, we were interested to determine if similar synergistic interactions occurred when using BV or non-BV G. vaginalis isolates. Surprisingly, with the exception of one species (M. mulieris), no differences were found between BV and non-BV associated $G$. vaginalis mediated dual-species biofilm augmentation. These results suggests that the key difference in BV or non-BV G. vaginalis virulence potential seems not to be related with biofilm maturation, at least in a dual-species model. We propose that once specific strains of $G$. vaginalis are able to outcompete the resident Lactobacillus species and start to growth in clusters, secondary anaerobes will easily incorporate the biofilm. This might be the key difference in virulence potential of $G$. vaginalis [9].

A particular example of synergistic interaction in dual-species biofilms is the case of $G$. vaginalis and P. bivia. It has been previously shown that $G$. vaginalis produces amino acids through its metabolism and $P$. bivia, a strict anaerobe, uses amino acids as its fuel source and as a result produces ammonia, which in turn is used by G. vaginalis [14]. Nevertheless, our data also showed that L. vaginalis had an antagonistic effect in the presence of all tested G. vaginalis biofilms. Boskey and colleagues have showed that the 


Strains ( $\mathbf{n}=\mathbf{2 4 )}$
Actinomyces neuii
Brevibacterium ravenspurgense
Corynebacterium amycolatum
Corynebacterium tuscaniense
Staphylococcus hominis
Staphylococcus saprohyticus
Enterococcus faecalis
Nosocomiicoccus ampullae
Staphylococcus simulans
Staphylococcus warnerii
Streptococcus anginosus
Propionibacterium acnes
Escherichia coli
Bacillus firmus
Prevotella bivia
Staphylococcus haemolyticus
Streptococcus agalactiae
Actinomyces turicensis
Gemella haemolysans
Aerococcus christensenii
Corynebacterium tuberculostearicum
Staphylococcus epidermidis
Mobiluncus mulieris
Lactobacillus vaginalis

Synergistic interaction

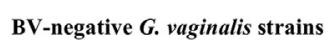

UM131 UM085 UM246

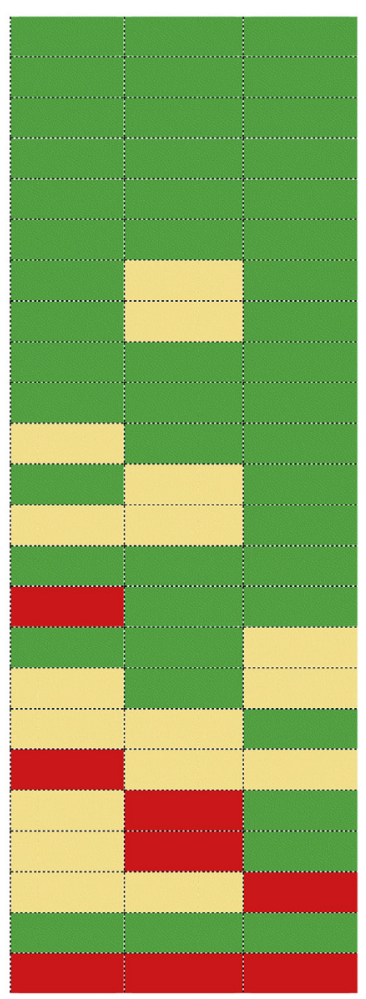

BV-positive $G$. vaginalis strains

UM241 UM121 UM137

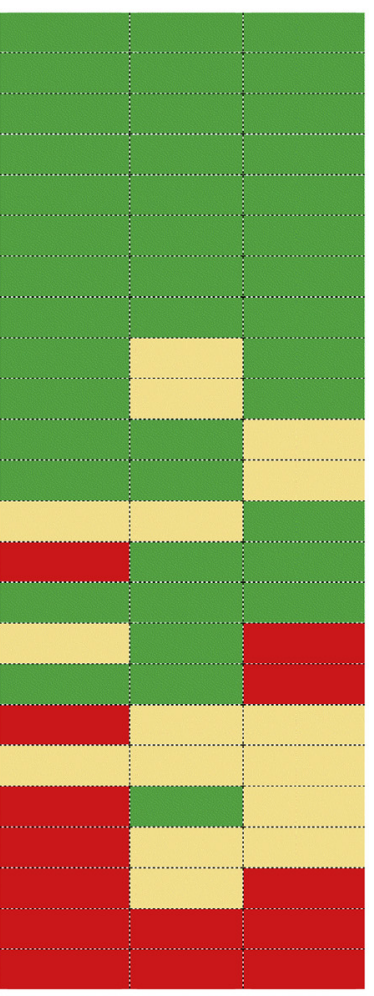

Neutral interaction

Antagonistic interaction

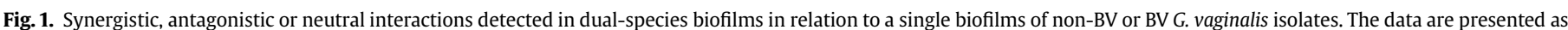

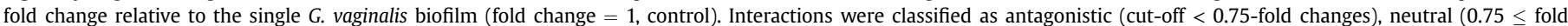

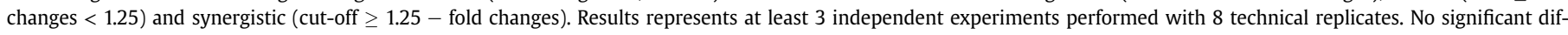

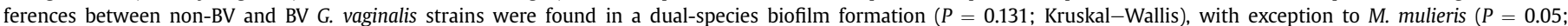
Kruskal-Wallis).

growth limiting factor for $L$. vaginalis was a depletion of a metabolite or the buildup of an unspecified toxic waste product [15], that might also be toxic to G. vaginalis causing a disruption of the biofilm. Curiously, our findings revealed that $M$. mulieris was the only bacterial specie with opposing interactions in the presence of either non-BV or BV pre-formed G. vaginalis biofilms. Nevertheless, further work is required to explore the bacterial interactions between these bacterial species.

The results from our study should be interpreted in light of several limitations. First, initial adhesion by G. vaginalis was performed in polystyrene microtiter plate wells rather than vaginal epithelium, where the presence of host-derived factors (e.g. mucus production, specific receptors on the epithelial surface) can influence bacterial adherence and biofilm formation. This technical limitation is not easy to overcome since, as we shown before, G. vaginalis quickly induces cytotoxic changes and detachment of pre-adhered epithelial cultures [9]. Furthermore, the growth medium did not contain all of the factors found in vivo, and some in vivo cues may turn on expression of biofilm-related genes. However, these limitations aside, in vitro models can be very informative and are key to furthering our understanding on multispecies biofilms and the development of BV.

In conclusion, this study provides direct evidence that confirms synergistic roles of many secondary or late colonizers in BV multi- species biofilm development, but reveals that those interactions are not specific for more virulent BV-associated G. vaginalis.

\section{Acknowledgements}

The authors thank the FCT Strategic Project of UID/BIO/04469/ 2013 unit and the project RECI/BBB-EBI/0179/2012 (FCOMP-010124-FEDER-027462). The funders had no role in study design, data collection and analysis, decision to publish, or preparation of the manuscript. JC was funded by the FCT individual fellowship SFRH/ $\mathrm{BD} / 93963 / 2013$. NC is an Investigador FCT.

\section{References}

[1] J.R. Schwebke, C.A. Muzny, W.E. Josey, Role of Gardnerella vaginalis in the pathogenesis of bacterial vaginosis: a conceptual model, J. Infect. Dis. 210 (2014) 338-343. http://dx.doi.org/10.1093/infdis/jiu089.

[2] A. Swidsinski, V. Loening-Baucke, W. Mendling, Y. Dörffel, J. Schilling Z. Halwani, et al., Infection through structured polymicrobial Gardnerella biofilms (StPM-GB), Histol. Histopathol. 29 (2013) 567-587, http://dx.doi.org/ 10.14670/HH-29.10.567.

[3] J.M. Marrazzo, Interpreting the epidemiology and natural history of bacterial vaginosis: are we still confused? Anaerobe 17 (2011) 186-190, http:// dx.doi.org/10.1016/j.anaerobe.2011.03.016.

[4] M.J. Redelinghuys, M.M. Ehlers, A.W. Dreyer, M.M. Kock, Normal flora and bacterial vaginosis in pregnancy: an overview, Crit. Rev. Microbiol. (2015) 1-12, http://dx.doi.org/10.3109/1040841X.2014.954522. 
[5] B.M. Peters, M.A. Jabra-Rizk, G.A. O'May, J.W. Costerton, M.E. Shirtliff, Polymicrobial interactions: impact on pathogenesis and human disease, Clin. Microbiol. Rev. 25 (2012) 193-213, http://dx.doi.org/10.1128/CMR.00013-11.

[6] J.M. Marrazzo, L.A. Koutsky, D.A. Eschenbach, K. Agnew, K. Stine, S.L. Hillier Characterization of vaginal flora and bacterial vaginosis in women who have sex with women, J. Infect. Dis. 185 (2002) 1307-1313, http://dx.doi.org $10.1086 / 339884$.

[7] T.P. Jayaprakash, J.J. Schellenberg, J.E. Hill, Resolution and characterization of distinct cpn60-based subgroups of Gardnerella vaginalis in the vaginal microbiota, PLoS One 7 (2012) e43009. http://dx.doi.org/10.1371/journal. pone.0043009.

[8] P. Alves, J. Castro, C. Sousa, T.B. Cereija, N. Cerca, Gardnerella vaginalis outcompetes 29 other bacterial species isolated from patients with bacterial vaginosis, using in an in vitro biofilm formation model, J. Infect. Dis. 210 (2014) 593-596. http://dx.doi.org/10.1093/infdis/jiu131.

[9] J. Castro, P. Alves, C. Sousa, T. Cereija, Â. França, K.K. Jefferson, et al., Using an in-vitro biofilm model to assess the virulence potential of bacterial vaginosis or non-Bacterial vaginosis Gardnerella vaginalis isolates, Sci. Rep. 5 (2015) 11640. http://dx.doi.org/10.1038/srep11640.

[10] A. Swidsinski, W. Mendling, V. Loening-Baucke, A. Ladhoff, S. Swidsinski,
L.P. Hale, et al., Adherent biofilms in bacterial vaginosis, Obstet. Gynecol. 106 (2005) 1013-1123. http://dx.doi.org/10.1097/01.AOG.0000183594.45524.d2.

[11] A. Machado, K.K. Jefferson, N. Cerca, Interactions between Lactobacillus crispatus and bacterial vaginosis (BV)-Associated bacterial species in initial attachment and biofilm formation, Int. J. Mol. Sci. 14 (2013) 12004-12012, http://dx.doi.org/10.3390/ijms140612004.

[12] D. Machado, A. Palmeira-de-Oliveira, N. Cerca, Optimization of culture conditions for Gardnerella vaginalis biofilms formation, J. Microbiol. Methods 14 (118) (2015) 143-146, http://dx.doi.org/10.1016/j.mimet.2015.09.007.

[13] A. Machado, N. Cerca, Influence of biofilm formation by Gardnerella vaginalis and other anaerobes on bacterial vaginosis, J. Infect. Dis. (2015), http:// dx.doi.org/10.1093/infdis/jiv338 (in press).

[14] V. Pybus, A.B. Onderdonk, Evidence for a commensal, symbiotic relationship between Gardnerella vaginalis and Prevotella bivia involving ammonia: potential significance for bacterial vaginosis, J. Infect. Dis. 175 (1997) 406-413. http://dx.doi.org/10.1093/infdis/175.2.406.

[15] E.R. Boskey, K.M. Telsch, K.J. Whaley, T.R. Moench, R.A. Cone, Acid production by vaginal flora in vitro is consistent with the rate and extent of vaginal acidification, Infect. Immun. 67 (1999) 5170-5175. PMID: 10496892. 